\title{
Hyperperfect Numbers With Three Different Prime Factors
}

\section{By Herman J. J. te Riele}

\begin{abstract}
The existence of hyperperfect numbers with more than two different prime factors is shown by five examples.
\end{abstract}

Recently, Minoli [2] has defined $n$-hyperperfect numbers as positive integers $m$ such that there is some positive integer $n$ with

$$
m=1+n[\sigma(m)-m-1] .
$$

1-hyperperfect numbers are the classical perfect numbers. Minoli gives a list of all $n$-hyperperfect numbers $<1,500,000$ with $n>1$, and these numbers have the form $p^{\alpha} q$, where $p$ and $q$ are prime numbers, $p<q$ and $\alpha \in \mathbf{N}$. Minoli wonders whether all hyperperfect numbers might have this form. By using a well-known technique, which was used, for instance, by Euler [1] to compute amicable number pairs, we have computed five hyperperfect numbers, each with three different prime factors.

Let $m=p q r, p<q<r$ prime numbers, be an $n$-hyperperfect number. By (1) we have

$$
p q r=1+n(p q+p r+q r+p+q+r) .
$$

Now, if we assume that $p$ and $n$ are given, this is a quadratic equation in $q$ and $r$. We write it as

$$
(p-n) q r-n(p+1) q-n(p+1) r=1+n p .
$$

Multiplying by $(p-n)$, and adding $n^{2}(p+1)^{2}$ to both sides yields

$$
\begin{array}{r}
{[(p-n) q-n(p+1)][(p-n) r-n(p+1)]} \\
=(p-n)(1+n p)+n^{2}(p+1)^{2} .
\end{array}
$$

If $A B, A<B$, is a factorization of the known right-hand side, then we can write

$$
q=[n(p+1)+A] /(p-n), \quad r=[n(p+1)+B] /(p-n) .
$$

If now both $q$ and $r$ are integers and prime, then $p q r$ is an $n$-hyperperfect number. Clearly, a small value of $(p-n)$ will facilitate finding integers $q$ and $r$. The simplest choice is $p-n=1$; this gives

(2) $q=p^{2}-1+A, \quad r=p^{2}-1+B, \quad A B=p^{4}-p^{2}-p+2, \quad A<B$.

If $A=1$, then $q=p^{2}$, not a prime. If $p \equiv 2(\bmod 3)$, then $p^{2}-1 \equiv 0(\bmod 3)$ and $A B=p^{4}-p^{2}-p+2 \equiv 0(\bmod 3)$, so that $3 \mid A$ or $3 \mid B$; hence, at least one of $q$ 
and $r$ is composite. Excluding these cases we have checked (2) for all primes $p<300$ (and $n=p-1$ ), and all possible factorizations $A B$. We found the following five hyperperfect numbers, each with three different prime factors:

$$
\begin{array}{rlrl}
1570153 & =13 \cdot 269 \cdot 449 & & n=12, \\
3675965445337 & =229 \cdot 67187 \cdot 238919 & & n=228, \\
8898807853477 & =283 \cdot 112087 \cdot 280537 & n & =282, \\
72315968283289 & =277 \cdot 78541 \cdot 3323977 & & n=276, \\
348231627849277 & =223 \cdot 49807 \cdot 31352557 & & n=222 .
\end{array}
$$

Remark. All $n$-hyperperfect numbers in Minoli's table with odd $n$ are instances of the following rule: if both $p=6 k-1$ and $q=12 k+1$ are prime numbers for some $k \in \mathrm{N}$, then $p^{2} q$ is an $n$-hyperperfect number with $n=4 k-1$. We conjecture that there are infinitely many hyperperfect numbers.

Note added in proof. By generalizing the technique described in this note, we have constructed seven more hyperperfect numbers (for details, see [3]):

$$
\begin{array}{rlrl}
60110701 & =7^{2} \cdot 383 \cdot 3203 & n=6, \\
13544168521 & =13^{2} \cdot 2347 \cdot 34147 & n=12, \\
8992165119733 & =19^{2} \cdot 6871 \cdot 3625243 & n=18, \\
217158581600773 & =43^{2} \cdot 84319 \cdot 1392883 & n=42, \\
7972299196816043329 & =97^{2} \cdot 913571 \cdot 927465611 & n=96, \\
3736320978727037068273 & =73^{3} \cdot 31293799 \cdot 306914431 & n=72, \\
1605108132959576124160002571981 & =1327 \cdot 6793 \cdot 10020547039 \cdot 17769709449589
\end{array}
$$

Mathematical Centre

Kruislaan 413

1098 SJ Amsterdam, The Netherlands

1. L. EulER, “De Numeris Amicabilibus", Leonhardi Euleri Opera Omnia, Teubner, Leipzig and Berlin, Ser. I, vol. 2, 1915, pp. 86-162.

2. D. Mnou, "Issues in nonlinear hyperperfect numbers," Math. Comp., v. 34, 1980, pp. 639-645.

3. H. J. J. TE RIELE, Hyperperfect Numbers With More Than Two Different Prime Factors, Report NW 87/80, Mathematical Centre, Amsterdam, August 1980. 